\title{
On CONSISTEnCY AND DECIDABILITy IN Some Paraconsistent ARIthmetics
}

\author{
Andrew Tedder \\ Department of Logic, Institute of Philosophy, The Czech Academy of Sciences
}

\begin{abstract}
The standard style of argument used to prove that a theory is undecidable relies on certain consistency assumptions, usually that some fragment or other is negation consistent. In a non-paraconsistent setting, this amounts to an assumption that the theory is non-trivial, but these diverge when theories are couched in paraconsistent logics. Furthermore, there are general methods for constructing inconsistent models of arithmetic from consistent models, and the theories of such inconsistent models seem likely to differ in terms of complexity. In this paper, I begin to explore this terrain, working, particularly, in inconsistent theories of arithmetic couched in three-valued paraconsistent logics which have strong (i.e. detaching) conditionals.
\end{abstract}

\section{Introduction}

The tradition of relevant and paraconsistent arithmetic, following the pioneering work of Robert K. Meyer in [11, 12, 13], has produced many noteworthy results. Among the most interesting of these have concerned inconsistent models of arithmetic. The investigation into such structures, starting in Meyer's work and further driven by Dunn's [6] remarkable result concerning three-valued model theory has led to a variety of interesting developments in inconsistent model theory; see $[14,15,21,22,17,18,7]$. Many of the noteworthy results are consequences of the fact that Peano Arithmetic $\mathcal{P} \mathcal{A}$, formulated in Priest's three-valued paraconsistent logic LP, has finite, and

Australasian Journal of Logic (18:5) 2021, Article no. 11 
hence decidable, inconsistent models. ${ }^{1}$ This remains true when $\mathbf{L P}$ is extended by a primitive conditional connective obeying the rule modus ponens (I'll call such a connective a detaching conditional), as in the logic A3, against the background of which some arithmetic theories are studied in [28]. The existence of decidable extensions of $\mathcal{P} \mathcal{A}$ in paraconsistent logic raises interesting questions concerning the relationship between decidability, consistency, and related properties of theories.

In particular, a widely studied property of theories couched in classical logic is essential undecidability - informally put, a theory is essentially undecidable just when neither it nor any of its (consistent) extensions are decidable. The reason for the parenthetical word in the previous sentence is that a trivial theory is decidable whenever it is formulated in a recursively defined language, and triviality and inconsistency collapse in a non-paraconsistent setting, such as that of classical logic. Among theories which are essentially undecidable when formulated in classical logic, some of the most famous are two weak arithmetics: Robinson's arithmetic $\mathcal{Q}$ and Robinson's other arithmetic $\mathcal{R}$. While $\mathcal{Q}$ is, almost certainly, more famous (e.g. it has come up for relevant appraisal [5]), in some respects $\mathcal{R}$ is more interesting. In particular, it is perhaps the weakest essentially undecidable arithmetic which is still natural, and, as shown in [3], it remains essentially undecidable in a large class of logics including classical, intuitionist, fuzzy, and some substantially weaker non-classical logics. In fact, what was shown in [3] is that $\mathcal{R}$ has the separation property (according to the terminology used there, $\mathcal{R}$, formulated in a rather weak logic $\mathbf{L}_{0}$, is a Rosser theory) and this fact is used to prove essential undecidability following an argument form due to Kleene. ${ }^{2}$ The definition of essentially undecidable theory given in [3] is more general than usual, since it requires that any extension of the theory formulated in any extension of the logic be undecidable. However, the generalisation only goes so far, and the definition still only requires the undecidability of $\Sigma_{1}$-consistent extensions of $\mathcal{R}$ in $\mathbf{L}_{0}$. In a paraconsistent setting, where we can have interesting, non-trivial, inconsistent (perhaps even $\Sigma_{1}$-inconsistent) extensions

\footnotetext{
${ }^{1}$ Most of the models, logics, and theories of interest here have (more or less) standard names consisting of alpha-numeric strings. To distinguish I'll use a $\mathcal{C}$ alligraphic font for names of theories, Bold-face for the names of logics, and Fraktur for the names of particular models (I'll use $M$ as a metavariable over models when speaking generally).

${ }^{2}$ From this it is also shown that in a slightly smaller class of logics, those extending a version of the non-distributive Lambek calculus with thinning and explosion, this result also entails that $\mathcal{Q}$ is essentially undecidable.
}

Australasian Journal of Logic (18:5) 2021, Article no. 11 
of a theory, it would seem that the decidability properties of these is worth investigating.

In this paper, I'll prove some results concerning $\mathcal{Q}$ and $\mathcal{R}$ in two paraconsistent logics with detaching conditionals, particularly as concerns their (un)decidability, and that of their extensions. One such result applies the argument form given in [3], while the others indicate how various kinds of inconsistent models, and the theories of those models, complicate the usual situation as concerns decidability. Many of these results are variations on results existing in the literature, but I will present some new proofs concerning the relationship between $\mathcal{Q}$ and $\mathcal{R}$, and furthermore aim here to catalogue the old and new results in a way suggesting avenues for more systematic investigations into this topic in the future. I start (\$2) with preliminaries: namely model-theoretic characterisations of, first, the key logics LP, RM3, and $\mathbf{A 3}$, and then the most important kinds of inconsistent model, alongside some standard results about these. The bulk of the paper (§3) proceeds as follows: first I investigate the relationship between strong paraconsistent versions of $\mathcal{Q}$ and $\mathcal{R}$, showing that the latter is stronger than the former in the sense that the finite collapse models of $[21,17]$ are not models of it; then I show that a minor expansion of $\mathcal{R}$, closed under $\mathbf{A} \mathbf{3}$, is essentially undecidable in the sense of, and using the proof method of, [3]; finally, I'll discuss in more detail two models of interest for some open problems. The first model, due to Sirokofskich and Paris [24, 18], has a decidable theory in $\mathbf{L P}$, and I'll present some reason to think its theory in $\mathbf{A} \mathbf{3}$ is also decidable. After this, I'll discuss a class of infinite inconsistent models in which the true contradictions all concern 'non-standard numbers', and give some reasons to think that the A3 theory of such models will be inconsistent, infinite, nontrivial, and undecidable. In the appendix I'll give axiomatic formulations of RM3 and A3.

\section{Preliminaries}

Definition 2.1 (Languages). A language signature is composed of a set Con of name constants, a set Fun of functions (each with a fixed arity in the natural numbers), and a set Pred of predicates (of fixed arities). The language of arithmetic (with no subtraction or division) will be my focus, referred to as $\mathcal{L}$, and its signature consists in Con $=\{\overline{0}\}$, Fun $=\left\{S^{1},{ }^{2},{ }^{2}\right\}$, 
and Pred $=\left\{=^{2}, \leq^{2}\right\}$ where the superscripts denote arity. ${ }^{3}$ In this signature, let the set of numerals contain $\overline{0}$ and $\bar{n}:=\underbrace{S \ldots S}_{n}(\overline{0})$ for each $n \in \mathbb{Z}^{+}$.

In addition to the signature, a language is composed of a denumerable set of variables Var, a set of terms Term defined out of Var, Con, Fun as usual, and formulas, defined from Term, Pred and connectives/quantifiers as usual. All logics here will include the connectives $\neg, \vee$ (with arities 1,2 respectively) and the quantifier $\forall$. Two defined connectives and a defined quantifier are as below:

- $A \wedge B:=\neg(\neg A \vee \neg B)$

- $A \supset B:=\neg A \vee B$

- $\exists x A:=\neg \forall x \neg A$

All the systems other than $\mathbf{L P}$ will also include a binary connective $\rightarrow$ (distinct from $\supset$ ). Finally, to simplify some formulas, I'll take $\rightarrow$ to be the weakest-binding connective (so, for instance, $(A \vee B) \rightarrow(C \wedge D)$ can be re-written $A \vee B \rightarrow C \wedge D)$.

\subsection{LP and Two 3-Valued Extensions}

The basic logic here is Priest's LP, presented in [19].

Definition 2.2 (LP Model). An LP model is a tuple $M=\langle D, I\rangle$ where $D \neq \varnothing$ and $I$ is such that:

- If $c \in$ Con then $I(c) \in D$

- If $g \in F u n$ is $n$-ary then $I(g): D^{n} \longrightarrow D$

- If $R \in$ Pred is $n$-ary, $I(R)=\left\langle I^{+}(R), I^{-}(R)\right\rangle$ s.t. $I^{+}(R), I^{-}(R) \subseteq D^{n}$, and furthermore $I^{+}(R) \cup I^{-}(R)=D^{n}$.

\footnotetext{
${ }^{3}$ Note that identity does not have a fixed interpretation across all models, but will have its interpretation fixed w.r.t. to particular models. In fact, the truth conditions for identity statements will be common across the inconsistent models to be considered here, and it will be the falsity conditions which fluctuate.
}

Australasian Journal of Logic (18:5) 2021, Article no. 11 
Intuitively, $I^{+}(R)$ is the extension of $R$, comprising those (tuples of) objects of which $R$ is true, while $I^{-}(R)$ is the anti-extension, comprising those (tuples of) objects of which $R$ is false. The requirement that these exhaust the domain guarantees, in light of the truth conditions given below for $\neg$, that the law of excluded middle holds. ${ }^{4}$ For the next step, given $M$ and a variable assignment $\mathfrak{f}: \operatorname{Var} \longrightarrow D$, let $I_{\mathfrak{f}}:$ Term $\longrightarrow D$ be such that:

- if $\tau \in$ Con then $I_{\mathfrak{f}}(\tau)=I(\tau)$

- if $\tau \in \operatorname{Var}$ then $I_{\mathfrak{f}}(\tau)=\mathfrak{f}(\tau)$

- if $g \in$ Fun of arity $n$ and $\tau_{1}, \ldots, \tau_{n} \in$ Term, then $I_{\mathfrak{f}}\left(g\left(\tau_{1}, \ldots, \tau_{n}\right)\right)=$ $I(g)\left(I_{\mathfrak{f}}\left(\tau_{1}\right), \ldots, I_{\mathfrak{f}}\left(\tau_{n}\right)\right)$

An LP model $M$ with valuation $\mathfrak{f}$ is a function $M_{\mathfrak{f}}: \mathcal{L} \longrightarrow \wp(\{t, f\}) /\{\varnothing\}$, defined as follows (where $\mathfrak{f}^{\prime}(y)=\mathfrak{f}(y)$ for all $y \neq x$, call $\mathfrak{f}^{\prime}$ an $x$-variant on $\mathfrak{f}$ ):

- $t \in M_{\mathfrak{f}}\left(R\left(\tau_{1}, \ldots, \tau_{n}\right)\right) \Longleftrightarrow\left\langle I_{\mathfrak{f}}\left(\tau_{1}\right), \ldots, I_{\mathfrak{f}}\left(\tau_{n}\right)\right\rangle \in I^{+}(R)$

- $f \in M_{\mathfrak{f}}\left(R\left(\tau_{1}, \ldots, \tau_{n}\right)\right) \Longleftrightarrow\left\langle I_{\mathfrak{f}}\left(\tau_{1}\right), \ldots, I_{\mathfrak{f}}\left(\tau_{n}\right)\right\rangle \in I^{-}(R)$

- $t \in M_{\mathfrak{f}}(\neg A) \Longleftrightarrow f \in M_{\mathfrak{f}}(A)$

- $f \in M_{\mathfrak{f}}(\neg A) \Longleftrightarrow t \in M_{\mathfrak{f}}(A)$

- $t \in M_{\mathfrak{f}}(A \vee B) \Longleftrightarrow t \in M_{\mathfrak{f}}(A) \cup M_{\mathfrak{f}}(B)$

- $f \in M_{\mathfrak{f}}(A \vee B) \Longleftrightarrow f \in M_{\mathfrak{f}}(A) \cap M_{\mathfrak{f}}(B)$

- $t \in M_{\mathfrak{f}}(\forall x A) \Longleftrightarrow t \in M_{\mathfrak{f}^{\prime}}(A)$ for all $x$-variants $\mathfrak{f}^{\prime}$ on $\mathfrak{f}$

- $f \in M_{\mathfrak{f}}(\forall x A) \Longleftrightarrow f \in M_{\mathfrak{f}^{\prime}}(A)$ for some $x$-variant $\mathfrak{f}^{\prime}$ on $\mathfrak{f}$

I'll write $M, \mathfrak{f} \vDash_{\mathbf{L P}} A$ just in case $t \in M_{\mathfrak{f}}(A)$, and $M \vDash A$ just in case $M, \mathfrak{f} \vDash_{\mathbf{L P}} A$ holds for any $\mathfrak{f}^{5}{ }^{5}$ Given a set of formulas $\mathcal{T}, M \vDash_{\mathbf{L P}} \mathcal{T}$ just in case for every $T \in \mathcal{T}, M \vDash_{\mathbf{L P}} T$. Finally $\Gamma \vDash_{\mathbf{L}} A$, for $\Gamma \cup\{A\} \subseteq \mathcal{L}$, holds iff for every model $M$, if $M \vDash_{\mathbf{L}} G$, for all $G \in \Gamma$, then $M \vDash_{\mathbf{L}} A$.

\footnotetext{
${ }^{4}$ In [3], we needed to extend the arithmetic theories to handle logics without excluded middle. Since LP, and the extensions given here, satisfy excluded middle, I'll work with the simpler versions of the theories here.

${ }^{5}$ Sometimes, when $A$ is a closed formula (i.e. with no free variables), I'll write the value of $A$ in $M$ just as $M(A)$.
}

Australasian Journal of Logic (18:5) 2021, Article no. 11 
From this definition, one can define the sets of models of two (language) extensions of $\mathbf{L P}$. The first, RM3, is the three-valued extension of the relevant logic $\mathbf{R}$ plus the mingle axiom. It has been widely studied, and information on it is available in $[1,23]$, among other places.

Definition 2.3 (RM3 Model). An RM3 model is like an LP model, but:

$$
M_{\mathfrak{f}}\left(A \rightarrow_{\mathbf{R M} 3} B\right)= \begin{cases}\{t, f\} & \text { if } M_{\mathfrak{f}}(A)=M_{f}(B)=\{t, f\} \\ \{t\} & \text { if } M_{\mathfrak{f}}(A)=\{f\} \text { or } M_{\mathfrak{f}}(B)=\{t\} \\ \{f\} & \text { else }\end{cases}
$$

Given an RM3 model $M$, the expressions " $M \vDash_{\mathbf{R M} 3} A$ " and " $M \vDash_{\mathbf{R M} 3} \mathcal{T}$ " are defined analogously to that given for $\mathbf{L P}$.

Less well known than RM3 is a strong paraconsistent logic A3, following my naming convention from [28]. This logic was first presented, as far as I'm aware, by D'Ottaviano and da Costa [4], and it was later studied by Avron [2]. This logic extends $\mathbf{L P}$ by a conditional very like the material implication, for which reason it is called "LP $+c m i$ " in [9].

Definition 2.4 (A3 Model). An A3 model is like an LP model, but:

$$
M_{\mathfrak{f}}\left(A \rightarrow_{\mathbf{A} 3} B\right)= \begin{cases}\{t\} & \text { if } M_{\mathfrak{f}}(A)=\{f\} \\ M_{\mathfrak{f}}(B) & \text { else }\end{cases}
$$

" $M \vDash_{\mathbf{A} \mathbf{3}} \mathcal{T}$ ", and similar expressions, are defined as before.

Where context clarifies which system is meant, I'll just write $\rightarrow$ or $\vDash$ without a subscript. In general, whenever a claim is made about the satisfaction of a theory in a model of a logic $\mathbf{L}$, the primitive implication should be interpreted as that belonging to $\mathbf{L}$.

Definition 2.5. Given a logic $\mathbf{L}$, among those considered here, and a theory $\mathcal{T} \subseteq \mathcal{L}$, let $\mathcal{T}_{\mathbf{L}}=\left\{A \mid \mathcal{T} \vDash_{\mathbf{L}} A\right\}$. Note that 'theory' is being used here just to refer to a set of sentences, with closure under a logic being applied 'after the fact', as it were. ${ }^{6}$

\footnotetext{
${ }^{6}$ Of course, this definition does imply that $\mathcal{T}_{\mathbf{L}}$ is a 'theory' in the more common use of the word (as given in [16, p. 21], for instance) since if $A, B \in \mathcal{T}_{\mathbf{L}}$ then $A \wedge B \in \mathcal{T}_{\mathbf{L}}$ (at least for any $\mathbf{L}$ I'll consider here), and furthermore if $\{A\} \vDash_{\mathbf{L}} B$ (or if $\mathbf{L} \in\{\mathbf{R M} 3, \mathbf{A 3}\}$ and $\vDash_{\mathbf{L}} A \rightarrow B$ ) and $A \in \mathcal{T}_{\mathbf{L}}$ then $B \in \mathcal{T}_{\mathbf{L}}$.
}

Australasian Journal of Logic (18:5) 2021, Article no. 11 
Definition 2.6. Let $M$ be a model in logic $\mathbf{L}$, among those considered here. Let $M_{\mathbf{L}}=\left\{A \mid M \vDash_{\mathbf{L}} A\right\}$, i.e. let it be the complete theory of the model, relative to $\mathbf{L}$.

Definition 2.7. Let $\mathbf{L}$ be a logic and $\mathcal{T}$ a theory in the language $\mathcal{L}$, perhaps the $\mathbf{L}$-theory of some model of $\mathcal{L}$.

- $\mathcal{T}_{\mathbf{L}}$ is inconsistent iff there is an $A \in \mathcal{L}$ such that $A, \neg A \in \mathcal{T}_{\mathbf{L}}$.

- $\mathcal{T}_{\mathbf{L}}$ is trivial iff $\mathcal{T}_{\mathbf{L}}=\mathcal{L}$.

- $\mathcal{T}_{\mathbf{L}}$ is decidable iff it is decidable whether $A \in \mathcal{T}_{\mathbf{L}}$, for any formula $A$.

- $\mathcal{T}_{\mathbf{L}}$ is Rosser (or has the separation property) just in case for any disjoint pair of recursively enumerable $\alpha, \beta \subseteq \mathbb{N}$, there is a formula $A(x)$ such that for any $n \in \mathbb{N}$ :

- $n \in \alpha \Rightarrow A(\bar{n}) \in \mathcal{T}_{\mathbf{L}}$

- $n \in \beta \Rightarrow \neg A(\bar{n}) \in \mathcal{T}_{\mathbf{L}}$

Finally, instead of $A \in \mathcal{T}_{\mathbf{L}}$, I'll usually write $\mathcal{T}_{\mathbf{L}} \vdash A$.

Note that any trivial theory is inconsistent and decidable. In order to decide inclusion in a trivial theory, apply the algorithm "when you see a formula, say "yes"!".

\subsection{Two Robinson Arithmetics}

The results here will concern two arithmetic theories, both studied extensively by Raphael Robinson [26], which are axiomatised as follows (to obtain versions of these theories in the language of LP replace each instance of $\rightarrow$ with $\supset)$.

\subsubsection{Basic Theory of Identity}

Each theory I consider will be include the following axioms governing identity (though this will be a non-logical predicate).

$(=$ ref $) x=x$

Australasian Journal of Logic (18:5) 2021, Article no. 11 
$(=\operatorname{com}) x=y \rightarrow y=x$

$(=\operatorname{tran}) x=y \rightarrow(y=z \rightarrow x=z)$

(LLterm) $x=y \rightarrow t(x)=t(y)$

for any term $t$

(LLform) $x=y \rightarrow(A(x) \rightarrow A(y))$

\subsubsection{Robinson's Arithmetic: $\mathcal{Q}$}

This axiomatisation is given by Dunn [5] in studying a version couched in a relevant logic, for which the distinction between the two versions of $(\mathcal{Q} 9)$ is salient. I'll call $\mathcal{Q}$ that theory axiomatised with $(\mathcal{Q} 9)$ and $\mathcal{Q}^{a}$ that with $(\mathcal{Q} 9 a)$. Note, it is always assumed that $S,+, \cdot$ are functional.

$(\mathcal{Q} 0) x \leq y \leftrightarrow \exists z(x+z=y)$

(Q1) $x+\overline{0}=x$

$(\mathcal{Q} 2) x+S(y)=S(x+y)$

(Q3) $x \cdot \overline{0}=\overline{0}$

(Q4) $x \cdot S(y)=(x \cdot y)+x$

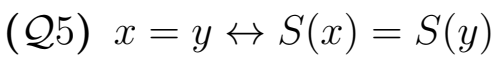

(Q6) $S(x) \neq \overline{0}$

(Q7) $x \neq \overline{0} \rightarrow \exists y(x=S(y))$

$(\mathcal{Q} 7 a) x \neq \overline{0} \supset \exists y(x=S(y))$

\subsubsection{Robinson's Other Arithmetic: $\mathcal{R}$}

$(\mathcal{R} 0) \quad x \leq y \leftrightarrow \exists z(x+z=y)$

(R1) $\bar{n}+\bar{m}=\overline{n+m}$

(R2) $\bar{n} \cdot \bar{m}=\overline{n \cdot m}$

(R3) $\bar{n} \neq \bar{m}$

for $n \neq m$

$(\mathcal{R} 4) x \leq \bar{n} \rightarrow(x=\overline{0} \vee \cdots \vee x=\bar{n})$

(R5) $x \leq \bar{n} \vee \bar{n} \leq x$

Classically, and in any logic extending the stronger system of [3], $\mathcal{R}$ is strictly weaker than $\mathcal{Q}$, and so essential undecidability results concerning the former can be used to obtain (essential) undecidability results concerning the latter.

Australasian Journal of Logic (18:5) 2021, Article no. 11 


\subsection{Some Models of Arithmetic}

For my purposes, some information is needed concerning a handful of different kinds of models of arithmetic. The first model is familiar.

Definition 2.8 (The Standard Model of Arithmetic). The standard model of arithmetic $N$ is that where formulas are assigned values in $\{\{t\},\{f\}\}$, and numerals and arithmetic operations are interpreted by the natural numbers $(\mathbb{N})$ and the usual operations thereon. Since $N$ assigns binary values, it is the classical standard model (as can be verified by checking the truth conditions on connectives in any of the logics under consideration here, noting that they assign the classical values when given classical inputs).

In addition to the standard model, I'll need to appeal to one more kind of classical model of arithmetic - for the purposes of discussing these models let $\mathbf{C L}$ be classical logic and $\vDash_{\mathbf{C L}}$ be classical satisfaction, defined as usual.

Definition 2.9 (Countable Non-Standard Models of Arithmetic). A model $M$ is a non-standard model of arithmetic is a model the initial segment of which is the natural numbers $\mathbb{N}$ (or any structure of that order type), followed by countably many copies of $\mathbb{Z}$ (or structures of that order type). For a more formal definition, and results concerning these, consult Kaye's classic book on models of Peano Arithmetic [10], or most textbooks on classical model theory. I'll only discuss them at an informal level, so will leave it here.

With these classical models out of the way, the more interesting models, for my purposes, are collapse models, which can be obtained from the standard models in a more or less fixed way:

Definition 2.10 (Collapse Models of Arithmetic). A collapse model of arithmetic is given by closing the structure of $N$ under a congruence relation $\sim$ on $\mathbb{N}$ (or an extension thereof by some non-standard numbers). The natural numbers (perhaps along with some non-standard numbers) are collected into equivalence classes according to $\sim$, and these are the elements of the collapsed model. That is, given $\sim$, set the equivalence class on $a \in \mathbb{N}$ to $[a]=\{b \in \mathbb{N} \mid a \sim b\}$ and 'type-lift' the arithmetic operations as follows: $S([x])=[S(x)],[x]+[y]=[x+y]$, and $[x] \cdot[y]=[x \cdot y]$.

A collapse model of arithmetic, in a logic extending $\mathbf{L P}$, consists in taking the quotient over $D$, either the natural numbers or an extension thereof by some non-standard numbers, $D_{\sim}=\{[a] \mid a \in D\}$, and $I$ such that, first,

Australasian Journal of Logic (18:5) 2021, Article no. 11 
$I(\overline{0})=[0]$, second, $S,+, \cdot$ satisfy the above listed constraints (i.e. so that $\sim$ is really a congruence w.r.t. these), and finally $=$ is as follows:

- $I^{+}(=)=\{\langle[a],[b]\rangle \mid a \sim b\}$

- $I^{-}(=) \supseteq\{\langle[a],[b]\rangle \mid a \neq b\}$

These equality conditions are where the action is, because while the extension of identity is fixed by the equivalence classes, the anti-extension, while required to include all pairs of unequal numbers, can be expanded beyond this. In fact, in all but one of the models I'll discuss, the Sirokofskich model, the $\supseteq$ will actually just be $=$. The general point here is that these conditions can generate inconsistent equality statements even between numerals, since two numbers may inhabit the same equivalence class, while being unequal to each other in the original model. In the Sirokofskich model one goes even farther by expanding the anti-extension of $=$ to include as many pairs as possible, making it so that almost all true equalities give rise to true contradictions, but I'll set aside further discussion of this model for later. As a final note, in these, and every other kind of model, I'll fix that, for any variable assignment $\mathfrak{f}, M_{\mathfrak{f}}(x \leq y)=M_{\mathfrak{f}}(\exists z(x+z=y))$, so this will be left tacit from here on out. ${ }^{7}$

I'll be interested in two kinds of finite collapse model here - on the left is a cyclic model with $n$ elements (i.e. a cycle with period $n$ ), $\mathfrak{C}^{n}$, and on the right is a heap model $\mathfrak{H}^{m, n}$ with a 'tail' of $m-1$ initial elements, followed by a cycle of period $n$ :
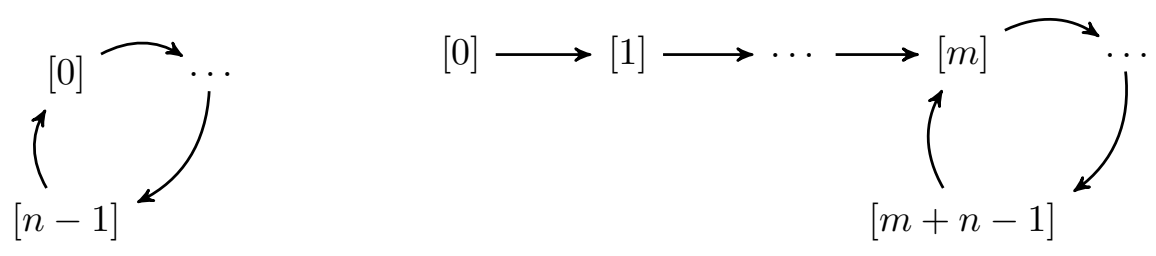

The following definitions rigorously characterise these kinds of models:

\footnotetext{
${ }^{7}$ Note that this is condition is sufficient to settle the truth of $(\mathcal{Q} 0)$ and $(\mathcal{R} 0)$ in the logics under consideration here.
}

Australasian Journal of Logic (18:5) 2021, Article no. 11 
- A cyclic model of arithmetic is induced by, fixing an $n \in \mathbb{N}$, setting $[x]=\{y \mid \exists k \in \mathbb{Z}(x+k n=y)\}$, and setting the anti-extension of $=$ to be $\{\langle[m],[n]\rangle \mid m \neq n\}$. Call $\mathfrak{C}^{n}$ that structure where $[n]=[0]$, i.e. that structure where $\sim$ is equivalence $\bmod n$.

- A heap model $\mathfrak{H}^{m, n}$ is induced by:

$$
[i]= \begin{cases}\{i\} & \text { if } i<m \\ \{j \mid \exists k \in \mathbb{Z}(i+k n=j)\} & \text { else }\end{cases}
$$

(with the anti-extension of $=$ as in the cyclic models).

Also of interest here is one special case not covered explicitly by the definition of $\mathfrak{H}$ - namely, where $m$ is allowed to be an infinite number. These are obtained by taking a countable, non-standard model and collapsing the nonstandard elements to form a cycle of period $m$. Call such a model $\mathfrak{H}^{\omega, m}$.

Let me record some immediate consequences of the definitions.

Proposition 2.11. Suppose $\boldsymbol{L} \in\{\boldsymbol{L P}, \boldsymbol{R} M 3, \boldsymbol{A} 3\}$ - then the following facts obtain:

(i) $\mathfrak{C}_{L}^{n}$ and $\mathfrak{H}_{L}^{m, n}$ are decidable for any $m, n \in \mathbb{N}$.

(ii) For $m_{1}, m_{2}<n$ and $m_{1} \neq m_{2}, \mathfrak{C}^{n} \not \nvdash_{\boldsymbol{L}} \overline{m_{1}}=\overline{m_{2}}$.

(iii) For $m_{1}, m_{2}<m$ and $m_{1} \neq m_{2}, \mathfrak{H}^{m, n} \not \nvdash_{\boldsymbol{L}} \overline{m_{1}}=\overline{m_{2}}$.

(iv) $\mathfrak{C}^{n} \vDash_{L} \bar{n}+\bar{n}=\bar{n} \wedge \bar{n}+\bar{n} \neq \bar{n}$.

(v) For $n>0, \mathfrak{H}^{m, n} \vDash_{\boldsymbol{L}} \bar{m}+\bar{n}=\bar{m} \wedge \bar{m}+\bar{n} \neq \bar{m}$.

These facts indicate that the finite models, in addition to being decidable, are also non-trivial and not only inconsistent but $\Delta_{0}$-inconsistent, as the inconsistency already features in (in)equalities between numerals. Lastly, I'll record the single most important fact about inconsistent models of arithmetic in $\mathbf{L P}$.

Australasian Journal of Logic (18:5) 2021, Article no. 11 
Lemma 2.12 (Collapse). Fix an $\boldsymbol{L P}$ model $M=\langle D, I\rangle$ in $\mathcal{L}$. Let $\sim$ be an equivalence relation on $D$ and a congruence on the interpretations of the function symbols, and let $I^{\sim}$ be defined from $I$ as indicated above, and set $M^{\sim}=\left\langle D^{\sim}, I^{\sim}\right\rangle$. Call the resulting model $M^{\sim}$. Then for any formula $A \in \mathcal{L}$, $M(A) \subseteq M^{\sim}(A)$ (i.e. if $t, f$ is in the value of $A$ in the original model, it is also in the value of $A$ in the collapsed model).

The proof of this result, and related results, are available in [21, 22], or pretty much anywhere fine inconsistent mathematics are sold. By way of explanation, in the language of arithmetic, in $\mathbf{L P}$ (i.e. with connectives $\vee, \neg$ ), the result of 'collapsing' a model under $\sim$ can only be to expand the truth values of the formulas. In particular, it cannot take a sentence from being satisfied to being not satisfied. The collapse lemma does not hold for A3 or RM3 since these contain detaching conditionals (this follows by a result of Ferguson [7, §13.3]) but nonetheless some three-valued models obtained by collapsing classical models are models of arithmetic theories closed under these logics. As we'll see, this fact has some interesting consequences for the question of decidable extensions in those paraconsistent arithmetics.

\section{Paraconsistent $\mathcal{Q}$ and $\mathcal{R}$}

First, I'll record some facts concerning versions of $\mathcal{R}$ and $\mathcal{Q}$ in A3 and RM3, indicating why Robinson's other arithmetic $\mathcal{R}$ is more interesting, in a paraconsistent setting with a detaching conditional. In particular, $\mathcal{R}$, in either logic, is more demanding than $\mathcal{Q}$ in the sense that it rules out a wider range of inconsistent models. The former rules out more inconsistent models than the latter. First, note that all cyclic models are models of $\mathcal{Q}_{\mathbf{A 3}}$ :

Theorem 3.1. For each $n \in \mathbb{N}, \mathcal{Q}_{A 3} \subseteq \mathfrak{C}_{A 3}^{n}$.

Proof. I'll leave the interested reader to verify that all arrow-free axioms of $\mathcal{Q}_{\mathrm{A} 3}$ hold in any cyclic model, so I'll only consider the arrow-including axioms. It is easy to see that $\mathfrak{C}_{\mathbf{A} 3}^{n} \vdash x=y \rightarrow S(x)=S(y)$, just given the definition of the type-lifted $S$. For the converse, note that if $\mathfrak{C}_{\mathrm{A} 3}^{n} \vdash S(x)=$ $S(y)$ then $\langle[S(x)],[S(y)]\rangle \in I_{\mathfrak{C}^{n}}^{+}(=)$and thus $\exists k \in \mathbb{N}(x+k n+1=y+1)$ from which it follows that $\exists k \in \mathbb{N}(x+k n=y)$ and so $\mathfrak{C}_{\mathbf{A} 3}^{n} \vdash x=y$. Thus $\mathfrak{C}_{\mathbf{A} 3}^{n} \vdash S(x)=S(y) \rightarrow x=y$. Similarly reasoning to the above guarantees that $\mathfrak{C}_{\mathbf{A} 3}^{n} \vdash x=y \rightarrow x+z=y+z \wedge z+x=z+y$. The argument for ( $\left.\mathcal{Q} 9\right)$ just 
holds due to the fact that $\mathfrak{C}_{\mathbf{A} 3}^{n} \vdash \exists y(x=S(y))$ holds for all $x$ and the fact that the A3 arrow satisfies thinning. As for $(\mathcal{Q} 0)$, this holds due to the assumption that, for any variable assignment $\mathfrak{f}, \mathfrak{C}_{\mathfrak{f}}^{n}(x \leq y)=\mathfrak{C}_{\mathfrak{f}}^{n}(\exists z(x+z=y)$ ) (from now on, steps like this one will be left tacit).

This leaves the identity axioms, of which I'll just consider (LLform), as the others are more or less immediate from the interpretation of $=$. Given the fact that every element of $\mathfrak{C}^{n}$ is named by a numeral, let us just consider the instance $\overline{m_{1}}=\overline{m_{2}} \rightarrow\left(A\left(\overline{m_{1}}\right) \rightarrow A\left(\overline{m_{2}}\right)\right.$. Note that, in any model $M$ of A3, $t \in M(B \rightarrow C)$ iff either $t \notin M(B)$ or $t \in M(C)$. So, in order to prove the desired result, it suffices to show that if $t \in \mathfrak{C}^{n}\left(\overline{m_{1}}=\overline{m_{2}}\right)$ and $t \in \mathfrak{C}_{\mathbf{A} \mathbf{3}}^{n}\left(A\left(\overline{m_{1}}\right)\right)$ then $t \in \mathfrak{C}_{\mathbf{A} \mathbf{3}}^{n}\left(A\left(\overline{m_{2}}\right)\right)$. This can be shown by induction on the complexity of $A$. The salient facts here are that, if $A$ is atomic, then we have the desired result simply because of the fact that $\sim$ is congruent over the arithmetic operations. Suppose that $A$ is a negated atomic formula - say, $A(x):=x=y$. If $t \in \mathfrak{C}_{\mathfrak{f}}^{n}\left(\overline{m_{1}} \neq y\right)$, and thus $f \in \mathfrak{C}_{\mathfrak{f}}^{n}\left(\overline{m_{1}}=y\right)$. Let us fix $\mathfrak{f}(y)=\left[m_{3}\right]$, and note that this entails that $\left\langle\left[m_{1}\right],\left[m_{3}\right]\right\rangle \in I_{\mathfrak{C}^{n}}^{-}(=)$, i.e. $m_{1} \neq m_{3}$. But by the assumption that $t \in \mathfrak{C}^{n}\left(\overline{m_{1}}=\overline{m_{2}}\right)$, it follows that $\left[m_{1}\right]=\left[m_{2}\right]$, and thus $\left\langle\left[m_{2}\right],\left[m_{3}\right]\right\rangle \in I_{\mathfrak{C}^{n}}^{-}(=)$. Thus $t \in \mathfrak{C}_{\mathfrak{f}}^{n}\left(\overline{m_{2}} \neq y\right)$, as desired. The other cases where $A(x)$ is a negated atomic are similar, so I'll leave them to the reader. This result amounts to showing that for any atomic formula, $A$, if $t \in \mathfrak{C}_{\mathfrak{f}}^{n}(x=y)$, then if $t \in \mathfrak{C}_{\mathfrak{f}}^{n}(A(x))$ then $t \in \mathfrak{C}_{\mathfrak{f}}^{n}(A(y))$ and if $f \in \mathfrak{C}_{\mathfrak{f}}^{n}(A(x))$ then $f \in \mathfrak{C}_{\mathfrak{f}}^{n}(A(y))$. The rest of the result follows by fixing this as the induction hypothesis: if $t \in \mathfrak{C}_{\mathfrak{f}}^{n}(x=y)$, then for any subformula $B$ of $A$, if $t / f \in \mathfrak{C}_{\mathfrak{f}}^{n}(B(x))$ then $t / f \in \mathfrak{C}_{\mathfrak{f}}^{n}(B(y)) .^{8}$

This has an immediate consequence related to decidability:

Corollary 3.2. $\mathcal{Q}_{\text {A3 }}$ has countably many extensions which are decidable, inconsistent, and non-trivial.

Proof. Immediately from Thm. 3.1 and Prop. 2.11, and the fact that each $\mathfrak{C}^{n}$ is finite, while all those with $n>1$ are non-trivial.

A related result is available for $\mathbf{R M} 3$, but only for $\mathcal{Q}^{a}$ therein:

\footnotetext{
${ }^{8}$ The reasoning to show that (LLform) is valid in the various other kinds of models I consider is similar to that here, so I'll leave it tacit in future arguments.
}

Australasian Journal of Logic (18:5) 2021, Article no. 11 
Proposition 3.3. While $\mathfrak{C}^{2} \not \nvdash_{\text {RM3 }} \overline{1} \neq \overline{0} \rightarrow \exists y(\overline{1}=S(y))$, nonetheless all the $\mathcal{Q}^{a}$ axioms are satisfied in each $\mathfrak{C}_{\boldsymbol{R} M 3}^{n}$.

Proof. Note that $\mathfrak{C}^{2}(\overline{1} \neq \overline{0})=\{t\}$ and yet $\mathfrak{C}^{2}(\overline{1}=S(\overline{2}))=\{t, f\}$, from which it follows that $\mathfrak{C}_{\mathbf{R M} 3}^{2}(\overline{1} \neq \overline{0} \rightarrow \exists y(\overline{1}=S(x)))=\{f\}$. The second part is verifiable by cases, with most of the cases the same as in Thm. 3.1.

This means we can obtain the following version of Cor 3.2 for RM3:

Corollary 3.4. $\mathcal{Q}_{\boldsymbol{R M}}^{a}$ has countably many decidable, inconsistent, and nontrivial extensions.

These propositions are interesting in light of the fact that, in general, $(\mathcal{R} 4)$ is not satisfied by cyclic models when interpreted using either of the detaching conditionals considered so far:

Lemma 3.5. For each $n \in \mathbb{N}, \mathfrak{C}^{n+2} \not \nvdash_{\boldsymbol{L}} x \leq \bar{n} \rightarrow(x=\overline{0} \vee \cdots \vee x=\bar{n})$ when $\boldsymbol{L} \in\{\boldsymbol{R} M 3, \boldsymbol{A} 3\}$.

Proof. Note that $\mathfrak{C}^{n+2}(\overline{n+1} \leq \bar{n})=\{t, f\}$ since $t \in \mathfrak{C}^{n+2}(\overline{n+1}+\overline{n+1}=\bar{n})$ because $2 n+2 \sim n$, and $f \in \mathfrak{C}^{n+2}(\overline{n+1}+\overline{n+1}=\bar{n}$ ) because $2 n+2 \neq n$ (similar reasoning provides that $f \in \mathfrak{C}^{n+2}(\overline{n+1}+\bar{m}=\bar{n})$ for any $\left.m \in \mathbb{N}\right)$. Hence $\mathfrak{C}^{n+2}(\exists y(\overline{n+1}+y=\bar{n}))=\{t, f\}$. On the other hand, $\mathfrak{C}^{n+2}(\overline{n+1}=\overline{0})=$ $\cdots=\mathfrak{C}^{n+2}(\overline{n+1}=\bar{n})=\{f\}$ because we have both $\overline{n+1} \nsim \overline{0}, \ldots, \overline{n+1} \nsim \bar{n}$ and $\overline{n+1} \neq \overline{0}, \ldots, \overline{n+1} \neq \bar{n}$. Thus by the definitions of $\rightarrow$ in either RM3 or A3, we have $\mathfrak{C}^{n+2}(\overline{n+1} \leq \bar{n} \rightarrow(\overline{n+1}=\overline{0} \vee \cdots \vee \overline{n+1}=\bar{n}))=\{f\}$.

This means that neither $\mathcal{Q}_{\mathbf{A} 3}$ nor $\mathcal{Q}_{\mathbf{R M} 3}^{a}$ satisfy $(\mathcal{R} 4)$, and thus:

Theorem 3.6. $\mathcal{R}_{A 3} \nsubseteq \mathcal{Q}_{A 3}$.

So in either logic, $\mathcal{R}$ is the 'stronger' theory in the sense that it can rule out finite cyclic models, while $\mathcal{Q}$ cannot. A further upshot of this for questions of decidability is that even if we can show that $\mathcal{R}$ is undecidable in A3, this fact doesn't automatically transfer over to $\mathcal{Q}$ (or $\mathcal{Q}^{a}$ ), as it does in many other logics, as in [3]. In fact, similar results can be given for finite heap models, which are also not models of $\mathcal{R}$ in A3 or RM3:

Lemma 3.7. $\mathfrak{H}^{m, n} \not \nvdash_{\boldsymbol{L}} \overline{m+1} \leq \bar{m} \rightarrow(\overline{m+1}=\overline{0} \vee \cdots \vee \overline{m+1}=\bar{m})$ when $\mathcal{L} \in\{\boldsymbol{A} 3, \boldsymbol{R} \boldsymbol{M} 3\}$ and $n>0$.

Australasian Journal of Logic (18:5) 2021, Article no. 11 
Of course, it can quickly be shown that finite heap models are not even models of $\mathcal{Q}$ or $\mathcal{Q}^{a}$ in A3 or RM3, because:

Lemma 3.8. $\mathfrak{H}^{m, n} \not \nvdash_{L} S(\overline{m-1})=S(\overline{m+n-1}) \rightarrow \overline{m-1}=\overline{m+n-1}$ when $\mathcal{L} \in\{\boldsymbol{A} 3, \boldsymbol{R} \boldsymbol{M} 3\}$.

and thus:

Theorem 3.9. $\mathcal{R}_{L}, \mathcal{Q}_{\text {RM3 }}^{a}, \mathcal{Q}_{\text {A3 }} \nsubseteq \mathfrak{H}_{\boldsymbol{L}}^{m, n}$ for $\boldsymbol{L} \in\{\boldsymbol{A} 3, \boldsymbol{R} \boldsymbol{M} 3\}$.

So of the finite models I've considered here, only the cyclic models tell the difference between paraconsistent $\mathcal{R}$ and $\mathcal{Q}$, when a detaching conditional is present, but nonetheless there is a substantial difference.

In fact, $\mathcal{R}$ is interesting in addition because the methods of [3] can be applied to show that a relatively minor extension of this theory in A3 (and a more major extension in RM3) is essentially undecidable.

\subsection{A Variation on Paraconsistent $\mathcal{R}$}

The aim in this section will be to prove that a certain extension of $\mathcal{R}_{\mathbf{A} 3}$ is $\Sigma_{1}$-complete, $\Sigma_{1}$-sound, and Rosser. This suffices to prove essentially undecidability. In order to adapt the proof of [3], I'll need the following 'classical' definition of the set of $\Sigma_{1}$-formulas:

Definition 3.10. A formula $A$ is a $\Delta_{0}$-formula $\left(A \in \Delta_{0}\right)$ iff $A$ is built up from atomic propositions by the use of $\wedge, \vee, \neg$ and bounded quantifiers of the following form, where $x \not \leq y$ is shorthand for $\neg(x \leq y)$ :

$$
\begin{aligned}
& \forall x \leq y A:=\forall x(x \not \leq y \vee A) \\
& \exists x \leq y A:=\exists x(x \leq y \wedge A)
\end{aligned}
$$

$A$ is a $\Sigma_{1}$-formula $\left(A \in \Sigma_{1}\right.$ ) iff $A$ has the form $\exists x B$ for some $B \in \Delta_{0}$.

$\mathcal{T}_{\mathbf{L}}$ is $\Sigma_{1}$-complete just in case for every $A \in \Sigma_{1}, N \vDash A \Rightarrow \mathcal{T}_{\mathbf{L}} \vdash A$.

$\mathcal{T}_{\mathbf{L}}$ is $\Sigma_{1}$-sound just in case for every $A \in \Sigma_{1}, \mathcal{T}_{\mathbf{L}} \vdash A \Rightarrow N \vDash A$.

First let me explain the need for an extension of $\mathcal{R}$ in these cases starting with $\mathcal{R}_{\mathbf{A} \mathbf{3}}$. While the implication logic of $\mathbf{A} \mathbf{3}$ is that of material implication, the full system does not admit the rule form of modus tollens: $A \rightarrow B, \neg B \Rightarrow \neg A$, (where $\Rightarrow$ should be understood as a separator in a rule of proof in Smiley's [25] sense). To see this, note that if $\mathbf{A} \mathbf{3}$ did admit this rule, one could quickly derive $(A \wedge \neg A) \rightarrow B$, which is not an A3 validity, from the following pair of A3 validities:

Australasian Journal of Logic (18:5) 2021, Article no. 11 
- $\neg((A \wedge \neg A) \rightarrow B) \rightarrow((A \wedge \neg A) \wedge \neg B)$

- $\neg((A \wedge \neg A) \wedge \neg B)$

In order to obtain a strong paraconsistent logic (i.e. one with a detaching conditional), the choice comes down to a few logical principles one can eject, and in the context of extensions of LP the salient choice is between thinning (K), written axiomatically as $A \rightarrow(B \rightarrow A)$ and contraposition (the axiom form of which is $(A \rightarrow B) \rightarrow(\neg B \rightarrow \neg A))$. A3 opts for $(\mathrm{K})$ and the above result indicates, in light of this fact, that even the rule form of modus tollens, (rMT), a very weak form of contraposition, is inadmissible in A3. ${ }^{9}$

The reason this fact is important is that one step of one of the lemmata needed for the proof of essential undecidability given in [3] requires its use (and I don't know of another way to do it). ${ }^{10}$ Besides (rMT), however, A3 has all the other properties needed for the argument structure followed in [3]. So the only necessary addition in order to obtain a theory for which that argument goes through would be to extend $\mathcal{R}$ to make up for the lack of (rMT). As a matter of fact, the argument of [3] only needs this rule in one place, so we can get away with adding one additional axiom scheme:

$$
\left({ }^{\prime}\right) \quad \neg(x=\overline{0} \vee \cdots \vee x=\bar{n}) \rightarrow x \not \leq \bar{n}
$$

Let $\mathcal{R}^{\prime}$ be the result of adding (') to $\mathcal{R}$. Note that this is not an additional logical axiom, but merely an arithmetic axiom, and one which seems innocuous (indeed, as we'll see, $\mathcal{R}_{\mathbf{A 3}}^{\prime}$ does still have models, and indeed inconsistent, non-trivial models, showing that the result is still paraconsistent). Also, since $\mathcal{R}_{\mathbf{A} 3}^{\prime}$ extends $\mathcal{R}_{\mathbf{A 3}}$, the non-inclusion results proved before also extend to the former. With this addition, we can make the argument form of [3] go through as follows - first proving a $\Sigma_{1}$-soundness and -completeness result, and then proving that $\mathcal{R}_{\mathbf{A 3}}^{\prime}$ is Rosser.

Lemma 3.11. $N \vDash_{C \boldsymbol{L}} A\left(\overline{t_{1}}, \ldots, \overline{t_{n}}\right) \Longleftrightarrow \mathcal{R}_{\boldsymbol{A} 3}^{\prime} \vdash A\left(\overline{t_{1}}, \ldots, \overline{t_{n}}\right)$, for $A \in \Sigma_{1}$.

\footnotetext{
${ }^{9}$ This fact also indicates that $\mathbf{A} \mathbf{3}$ is not congruent w.r.t. to valid biconditionals, since $A \leftrightarrow B$ can be valid while $\neg A \leftrightarrow \neg B$ is not; e.g. note that $(A \vee \neg A) \leftrightarrow(B \vee \neg B)$ is valid but $(A \wedge \neg A) \leftrightarrow(B \wedge \neg B)$ is generally not, despite the validity, in A3, of all DeMorgan equivalences.

${ }^{10}$ The fact that this rule is needed in [3] is somewhat hidden by the fact that they use a defined negation, from which the rule follows as a special case of the weak transitivity rule $A \rightarrow B, B \rightarrow C \Rightarrow A \rightarrow C$.
}

Australasian Journal of Logic (18:5) 2021, Article no. 11 
Proof. The right-to-left direction follows given that $\mathcal{R}_{\mathbf{A} 3}^{\prime}$ is a sub-theory of $\mathcal{R}_{\mathbf{C L}}$. For the converse, I'll adapt the proof of [3, Thm. 4.8]. The cases in which no negations occur go through just the same way in A3 as they do there, as the positive fragment of $\mathbf{A} \mathbf{3}$ properly extends that of the basic logic employed there (see the axiomatisation of A3 in the Appendix). So I'll just concern myself with those cases where $\neg$ occurs.

Suppose $A=\forall x \leq y B(x)$, and so $N \vDash_{\mathbf{C L}} B(\bar{m})$ for each $m \leq k$ (for fixed $k$ ). So by IH, it follows that $\mathcal{R}_{\mathbf{A} 3}^{\prime} \vdash B(\bar{m})$, and thus $\mathcal{R}_{\mathbf{A} 3}^{\prime} \vdash y=\bar{m} \rightarrow B(y)$. Since this holds for each such $m$, it follows that $\mathcal{R}_{\mathbf{A} 3}^{\prime} \vdash(y=\overline{0} \vee \cdots \vee y=$ $\bar{k}) \rightarrow B(y)$, so $\mathcal{R}_{\mathbf{A} 3}^{\prime} \vdash y \leq \bar{k} \rightarrow B(y)$, and thus $\mathcal{R}_{\mathbf{A} 3}^{\prime} \vdash y \leq \bar{k} \rightarrow y \not \leq \bar{k} \vee B(y)$, and since $\mathcal{R}_{\mathbf{A} \mathbf{3}}^{\prime} \vdash y \leq \bar{k} \vee y \not \leq \bar{k}$, it follows that $\mathcal{R}_{\mathbf{A} \mathbf{3}}^{\prime} \vdash y \not \leq \bar{k} \vee B(k)$, and thus $\mathcal{R}_{\mathbf{A} 3}^{\prime} \vdash \forall x(y \not \leq x \vee B(x))$, as desired.

The case where $A$ is $\bar{n} \neq \bar{m}$ is immediate. Next, if $N \vDash_{\mathbf{C L}} \overline{n+k} \neq \bar{m}$ then $n+k \neq m$ and so $\mathcal{R}_{\mathbf{A} \mathbf{3}}^{\prime} \vdash \overline{n+k} \neq \bar{m}$ and thus $\mathcal{R}_{\mathbf{A} \mathbf{3}}^{\prime} \vdash \bar{n}+\bar{k} \neq \bar{m}$ by the instance $\overline{n+k}=\bar{n}+\bar{k} \rightarrow(\overline{n+k} \neq \bar{m} \rightarrow \bar{n}+\bar{k} \neq \bar{m})$ of (=prin). The case for multiplication is similar.

The case which requires (') is that where $A$ is $\bar{n} \leq \bar{m}$. If $n \not \leq m$ then for each $k \leq n, k \neq m$, and thus for each such $k, \mathcal{R}_{\mathbf{A} 3}^{\prime} \vdash \bar{k} \neq \bar{m}$, and so $\mathcal{R}_{\mathbf{A} 3}^{\prime} \vdash \overline{0} \neq \bar{m} \wedge \cdots \wedge \bar{n} \neq \bar{m}$, so by (DeM), $\mathcal{R}_{\mathbf{A} 3}^{\prime} \vdash \neg(\overline{0}=\bar{m} \vee \cdots \vee \bar{n}=\bar{m})$, and thus $\mathcal{R}_{\mathrm{A} 3}^{\prime} \vdash \bar{n} \not \mathbf{m}$.

The cases where $A$ is the negation of a conjunction or disjunction formula follow from (DeM) in its various guises, and (DNE) takes care of the case where $A$ is a negation formula. The quantifier cases are similarly straightforward to adapt, given that $\forall x \neg A \rightarrow \neg \exists x A$ and $\exists x \neg A \rightarrow \neg \forall x A$ are valid in $\mathbf{A 3}$, and are left to the skeptical reader.

Lemma 3.12. $\mathcal{R}_{\boldsymbol{A} 3}^{\prime}$ is Rosser.

Proof. Consult the proof of [3, Thm. 4.9]. Altering notation a bit, the argument proceeds by starting with the assumption that $\alpha, \beta \subseteq \mathbb{N}$ are disjoint and r.e., and so that there are $\Delta_{0}$ formulas $A, B$ s.t.:

- $n \in A \Longleftrightarrow N \vDash_{\mathbf{C L}} \exists v A(\bar{n}, v)$

- $n \in B \Longleftrightarrow N \vDash_{\mathbf{C L}} \exists v B(\bar{n}, v)$

Given the definitions of $C(x, v):=\neg(\neg A(x, v) \vee \exists u \leq v B(x, u))$ and $D(x):=$ $\exists v C(x, v)$, the argument of [3] can be easily adapted to show $n \in A$ only if 
$\mathcal{R}_{\mathbf{A} 3}^{\prime} \vdash D(\bar{n})$ and $n \in B$ only if $\mathcal{R}_{\mathbf{A} 3}^{\prime} \vdash \neg D(\bar{n})$. The reason is that, since the (rMT) is not needed in that proof, every inference needed is available in $\mathcal{R}_{\mathrm{A} 3}$.

From these lemmata, the following shortly follows:

Theorem 3.13. Let $\boldsymbol{L}$ be an extension of $\boldsymbol{A} 3$, and let theory $\mathcal{T}$, in a language which extends the language of arithmetic, be such that $\mathcal{T}_{\boldsymbol{L}}$ is $\Sigma_{1}$-consistent and $\mathcal{R}_{\boldsymbol{L}}^{\prime} \subseteq \mathcal{T}_{\boldsymbol{L}}$. Then $\mathcal{T}_{\boldsymbol{L}}$ is undecidable.

A similar extension of RM3 can be used to prove an essential undecidability for an extension of $\mathcal{R}$ in RM3 - namely, one extended by certain instances of thinning. Actually, one can more or less immediately prove that:

Lemma 3.14. $\mathcal{R}_{R M 3}$ is $\Sigma_{1}$-sound and $\Sigma_{1}$-complete.

So the only place where the argument breaks down concerns the proof of Rosser-ness and the only inference needed in that argument which isn't available in RM3 is closure under an instance of the rule form of thinning, namely that if $\mathcal{R}_{\mathbf{L}} \vdash B(\bar{m})$ holds, where $B \in \Delta_{0}$, then $\mathcal{R}_{\mathbf{L}} \vdash \bar{m} \leq v \rightarrow B(\bar{m})$. So it's adequate to add to $\mathcal{R}$ axioms of the form (where $B$ may be any $\Delta_{0}$-formula containing some numeral $\bar{m}$, perhaps in addition to other terms):

$$
(*) \quad B(\bar{m}) \rightarrow(\bar{m} \leq v \rightarrow B(\bar{m}))
$$

obtaining $\mathcal{R}_{\mathbf{R M} 3}^{*}$. Then the arguments of [3] can be adapted to prove:

Lemma 3.15. $\mathcal{R}_{\boldsymbol{R} M 3}^{*}$ is Rosser.

and thus,

Theorem 3.16. Let $\boldsymbol{L}$ be an extension of $\boldsymbol{R} \boldsymbol{M} 3$, and let theory $\mathcal{T}$, in a language which extends the language of arithmetic, be such that $\mathcal{T}_{\boldsymbol{L}}$ is $\Sigma_{1}$ consistent and $\mathcal{R}_{L}^{*} \subseteq \mathcal{T}_{\boldsymbol{L}}$. Then $\mathcal{T}_{\boldsymbol{L}}$ is undecidable.

However this addition to $\mathcal{R}_{\mathbf{R M} 3}$ strikes me as much less innocuous than the addition needed for $\mathcal{R}_{\mathbf{A 3}}$. Perhaps most noteworthy here is that the schematicity required concerns not just terms but also formulas - it seems that we need to add very many instances of thinning. These provide some 
reasons to be skeptical of how minor an addition to $\mathcal{R}_{\mathbf{R M} 3}$ is needed in order to obtain this result.

In any case, for the rest of the paper I'll consider some further extensions of $\mathcal{R}_{\mathbf{A} 3}^{\prime}$, and pose some open problems concerning particular models. The overarching question here is, given that $\mathcal{R}_{\mathbf{A 3}}^{\prime}$ is essentially undecidable, what is the status of its inconsistent extensions? We've already seen that the finite collapse models do not satisfy even the $\mathcal{R}_{\mathbf{A 3}}$ axioms, since they fail to satisfy $(\mathcal{R} 4)$, which raises the question whether $\mathcal{R}_{\mathbf{A} 3}^{\prime}$ has any non-trivial decidable extensions - since the theorem indicates that none of its consistent extensions are decidable, these will have to be inconsistent extensions. Furthermore, does it have any undecidable, non-trivial, inconsistent extensions? While I won't answer these questions, I will point at likely candidate extensions (one which I suspect to be decidable, and one I suspect to be undecidable) and gesture at some facts which may provide some grist for the salient proofs.

\subsection{The Sirokofskich Model}

Sirokofskich and Paris $[24,18]$ produce an especially interesting (or perhaps especially uninteresting) inconsistent LP model of arithmetic - one which is both infinite and decidable. The trick is to make the model satisfy as many contradictions as possible, while still being non-trivial. Their model is defined as follows: fix $\mathcal{T}$, a complete, consistent extension of $\mathcal{P} \mathcal{A}_{\mathbf{C L}}$, and let $M$ be a countable non-standard model of $\mathcal{T}$. Define a sequence $\{0\}=C_{0} \subseteq$ $C_{1} \subseteq \cdots \subseteq C_{j} \subseteq \ldots$ of initial segments of $M$, closed under $S$ and , where $M=\bigcup C_{j}$. These initial cuts of the original model will form the elements of the collapsed model, for which purposes fix $a_{0}=0$ and $a_{j} \in C_{j} / C_{j-1}$ as representatives of their cuts (so, for example, the interpretation of $\overline{0}$ in $\mathfrak{S}, \mathfrak{S}(\overline{0})$ is $\left.\left[a_{0}\right]\right)$, and we'll fix the interpretation of successor so that $\left[a_{1}\right]$ is a successor of $\left[a_{0}\right]$, but otherwise every other element is its own successor, depicted as follows (where the arrows depict $S$, not $\leq$ ):

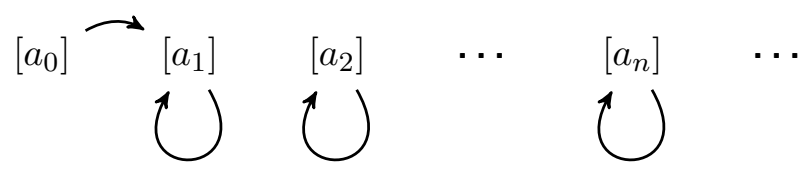

In order to get the desired behavour, one forms $\mathfrak{S}$ by closing $M$ under the congruence defined as follows:

Australasian Journal of Logic (18:5) 2021, Article no. 11 


$$
a \sim b \Longleftrightarrow \begin{cases}a=b=0 & \text { or } \\ a, b \in C_{j} / C_{j-1} \text { for some } j & \end{cases}
$$

So the domain of $\mathfrak{S}$ is $\left\{\left[a_{j}\right] \mid j \in \mathbb{N}\right\}$, and the type-lifted operations are defined as follows: $\left[a_{j}\right]+\left[a_{k}\right]=\left[a_{\max \{j, k\}}\right]$,

$$
S\left(\left[a_{j}\right]\right)=\left\{\begin{array}{ll}
{\left[a_{1}\right]} & \mathrm{j}=0 \\
{\left[a_{j}\right]} & \text { else }
\end{array} \quad\left[a_{j}\right] \cdot\left[a_{k}\right]= \begin{cases}{\left[a_{0}\right]} & \text { if } \min \{j, k\}=0 \\
{\left[a_{j}\right]+\left[a_{k}\right]} & \text { else }\end{cases}\right.
$$

Finally, $I_{\mathfrak{S}}^{-}(=)=M_{\sim} \times M_{\sim} /\left\langle\left[a_{0}\right],\left[a_{0}\right]\right\rangle$.

So successor is as described by the picture, addition just takes you to the larger of its addends, and multiplication behaves just like addition except in having zero as a multiplicative identity. As mentioned earlier, the trick here is to dramatically increase the number of true contradictions, by satisfying every negated identity except $\overline{0} \neq \overline{0}$, so that almost every true identity is a true contradiction.

Paris and Sirokofskich argue that $\mathfrak{S}_{\mathbf{L P}}$ is interpretable in classical Presburger arithmetic, and hence is decidable. They claim that for each formula $A$, one can recursively specify an $A^{*}$ s.t.:

$$
\mathfrak{S} \vDash_{\mathbf{L P}} A \Longleftrightarrow\langle\mathbb{N}, S,+,<,=\rangle \vDash_{\mathbf{C L}} A^{*}
$$

Since classical Presburger arithmetic is known to be decidable, this means that $\mathfrak{S}_{\mathbf{L P}}$ is also decidable. This has the remarkable result that $\mathfrak{S}_{\mathbf{L P}}$ is an inconsistent, non-trivial, and decidable theory of a model with an infinite domain. This suggests that the Sirokofskich model may also provide the means for finding a decidable extension of $\mathcal{R}_{\mathbf{A} 3}^{\prime}$. Indeed, it's A3-theory is an extension thereof:

Lemma 3.17. $\mathcal{R}_{A 3}^{\prime} \subseteq \mathfrak{S}_{A 3}$.

Proof. $(\mathcal{R} 1)$ and $(\mathcal{R} 2)$ are trivial, for if $n, m \neq 0, \bar{n}+\bar{m}=\bar{n} \cdot \bar{m}=\bar{n}=\bar{m}$ are all true in $\mathfrak{S}_{\mathbf{A 3}}$ - indeed, the interpretation of every numeral beside $\overline{0}$ is $\left[a_{1}\right]$ - the rest is obvious. Furthermore, the only negated equation which is not true in $\mathfrak{S}$ is $\overline{0} \neq \overline{0}$. This fact makes it clear that $\mathfrak{S}$ satisfies $(\mathcal{R} 3)$.

Let's move on to $(\mathcal{R} 4)$. Suppose that $t \in \mathfrak{S}(x \leq \bar{n})$. By the above, $\mathfrak{S}(\bar{n})=\left[a_{1}\right]$, so then for $x \leq \bar{n}$ to be true, fixing $\mathfrak{S}(x)=\left[a_{i}\right]$, it must be that 
there is an $a_{j}$ s.t. $\left[a_{i}+a_{j}\right]=\mathfrak{S}(\bar{n})$, i.e. $\left[a_{i}+a_{j}\right]=\left[a_{1}\right]$. Thus $\max \{i, j\}=1$, in which case either $a_{i}=a_{1}$ or $a_{i}=a_{0}$, and so $t \in \mathfrak{S}(x=\overline{0} \vee x=\overline{1})$ immediately.

The case for $(\mathcal{R} 5)$ is equally simple: note that if $\mathfrak{S}(x)=\left[a_{0}\right]$, then $\mathfrak{S}(x)+$ $\left[a_{1}\right]=\left[a_{1}\right]=\mathfrak{S}(\bar{n})$, and so $t \in \mathfrak{S}(\exists y(x+y=\bar{n}))$. Furthermore, if $\mathfrak{S}(x) \neq\left[a_{0}\right]$, then $\left[a_{1}\right]+\mathfrak{S}(x)=\mathfrak{S}(x)$, and so $t \in \mathfrak{S}(\exists y(\bar{n}+y=x))$.

This leaves just the verification of $\left(^{\prime}\right)$, which is also immediate.

In order to obtain a proof that $\mathfrak{S}_{\mathbf{A 3}}$ is decidable, what remains is to extend this translation to $\rightarrow_{\mathbf{A} 3}$. It seems likely that we can use $(A \rightarrow B)^{*}:=$ $A^{*} \supset B^{*}$, for the reason that for any $\mathbf{A} \mathbf{3}$ model, we have that for all $\mathfrak{f}$, $M_{\mathfrak{f}} \vDash_{\mathbf{A} 3} A \rightarrow B \Longleftrightarrow M \mathfrak{f} \nvdash_{\mathbf{A} 3} A$ or $M_{\mathfrak{f}} \vDash_{\mathbf{A} 3} B$. While this fact does not make $A \rightarrow B$ equivalent to $A \supset B$ in $\mathbf{A} \mathbf{3}$, we do have that the following are equivalent, when $A, B$ do not contain further conditionals (and no free variables, so we can avoid dealing with variable assignments):

- $\mathfrak{S} \vDash_{\mathbf{A} 3} A \rightarrow B$

- $\mathfrak{S} \nvdash_{\mathbf{A} 3} A$ or $\mathfrak{S} \vDash_{\mathbf{A} 3} B$

- $\langle\mathbb{N}, S,+,<,=\rangle \nvdash_{\mathbf{C L}} A^{*}$ or $\langle\mathbb{N}, S,+,<,=\rangle \vDash_{\mathbf{C L}} B^{*}$

- $\langle\mathbb{N}, S,+,<,=\rangle \vDash_{\mathrm{CL}} A^{*} \supset B^{*}$

Having noted this, I don't know whether this fact can be used to prove that $\mathfrak{S}_{\mathbf{A 3}}$ is also interpretable in classical Presburger arithmetic, for the reason that difficulties seem to arise in the case of universally quantified conditional formulas, but nonetheless it seems likely to me that:

Conjecture 3.18. $\mathfrak{S}_{\boldsymbol{A} 3}$ is interpretable in classical Presburger arithmetic.

If this were the case, it would, as with $\mathfrak{S}_{\mathbf{L P}}$, prove that $\mathfrak{S}_{\mathbf{A} 3}$ is decidable.

$\mathfrak{S}_{\mathbf{A} 3}$ is, in a sense, a rather simple model of arithmetic, in that the arithmetic functions usually behave either as an identity function or as a max function. To underline the way in which the model is simple, I'll set out some plausible axioms for the theory indicating its distinctive features. First, let's enrich the language $\mathcal{L}$ with a denumerable set $\left\{\hat{a}_{0}, \hat{a}_{1}, \ldots, \hat{a}_{n}, \ldots\right\}$ of (hatted) constants, fixing $\mathfrak{S}\left(\hat{a}_{j}\right)=\left[a_{j}\right]$. In giving some putative axioms below, I'll complicate matters by dealing both with numerals $\bar{n}$ and hatted constants, 
but this isn't a serious problem as all the non-zero numerals just cluster at $\left[a_{1}\right]$, so all could be rewritten to $\overline{1}$, if so desired.

Note that since $\mathfrak{S}\left(a_{0} \neq a_{0}\right)=\{f\}$, we can define a strong negation connective $A^{\perp}:=A \rightarrow \hat{a}_{0} \neq \hat{a}_{0}$. With strong negation, we can characterise the distinctive features of $\mathfrak{S}_{\mathbf{A 3}}$ in a term-schematic way (take any variables below as implicitly universally quantified):

Identity Principles

(A1) $\hat{a}_{0} \neq \hat{a}_{0} \rightarrow A$

(A2) $\left(\hat{a}_{j}=\hat{a}_{k}\right)^{\perp}$ when $j \neq k$

(A3) $\left(x=\hat{a}_{0}\right)^{\perp} \rightarrow x \neq y$

(A4) $\hat{a}_{0}=\overline{0}$

Successor Principles

(A5) $S\left(\hat{a}_{0}\right)=\hat{a}_{1}$

(A6) $\left(x=\hat{a}_{0}\right)^{\perp} \rightarrow S(x)=x$

(A7) $x=y \rightarrow S(x)=S(y)$

\section{Addition/Multiplication Principles}

(A8) $\hat{a}_{0}+x=x$

(A9) $\hat{a}_{0} \cdot x=\hat{a}_{0}$
(A10) $\hat{a}_{j}+\hat{a}_{k}=\hat{a}_{j}$
when $j \geq k$

(A11) $x=x+x=x \cdot x$

(A12) $x=y \rightarrow x+z=y+z \wedge z+x=z+y$

(A13) $x=y \rightarrow x \cdot z=y \cdot z \wedge z \cdot x=z \cdot y$ 
(A14) $\left(x=\hat{a}_{0}\right)^{\perp} \wedge\left(y=\hat{a}_{0}\right)^{\perp} \rightarrow x+y=x \cdot y$

(A15) $x+y=y+x, x \cdot y=y \cdot x$

First note that strong negation really is strong, i.e. $A^{\perp} \rightarrow \neg A$, given (A1), $(\mathrm{K}),(\mathrm{W})$, and $(\mathrm{B})$. Among the arithmetic consequences of these axioms is $\bar{n}=\hat{a}_{1}$ for all $n \in \mathbb{Z}^{+}$and thus $\bar{n}=\bar{m} \wedge \bar{n} \neq \bar{m}$ for all $n, m \in \mathbb{Z}^{+}$. So among

the consequences are formulas (or schemata) capturing the salient parts of the definition of the model, which suggests the following:

Conjecture 3.19. The above set of formulas axiomatises $\mathfrak{S}_{\boldsymbol{A}}$.

Given a proof of this, there may be another avenue to show that $\mathfrak{S}_{\mathbf{A} 3}$ is decidable, but I leave the question for future work.

\subsection{Infinite Heap Models of $\mathcal{R}_{\mathrm{A} 3}^{\prime}$}

There remains another interesting question in the area: are there any inconsistent, undecidable extensions of $\mathcal{R}_{\mathbf{A} 3}^{\prime}$ ? As before it seems that studying inconsistent models is of help here, and there are some reasonably natural candidates whose theories seem likely to be undecidable - the infinite heap models. While no finite heap model is a model of $\mathcal{R}_{\mathbf{A} 3}^{\prime}$, this is not the case for infinite ones. Indeed, unlike their finite neighbours, infinite heap models are even models of $\mathcal{Q}^{(a)}$ in A3 (RM3):

Theorem 3.20. For any $n \in \mathbb{N}, \mathcal{Q}_{A 3}^{a} \subseteq \mathfrak{H}_{\boldsymbol{A 3}}^{\omega, n}$ and $\mathcal{Q}_{\boldsymbol{R} M 3}^{a} \subseteq \mathfrak{H}_{\boldsymbol{R M}}^{\omega, n}$.

Proof. Note that the only counterexample to injectivity in the finite heap models occurs between the two immediate predecessors of the start of the cycle. However $\omega$ has no immediate predecessor and furthermore the cycle beginning with $\omega$ of period $n$ can produce no counterexamples either.

The argument concerning $\mathcal{Q}^{a}$ is similar to that before.

I'll go into more detail in the following proof, which is of more interest here:

Theorem 3.21. For each $m \in \mathbb{N}, \mathcal{R}_{A 3}^{\prime} \subseteq \mathfrak{H}_{\boldsymbol{A 3}}^{\omega, m}$. 
Proof. By the definition of $\sim$ for any inconsistent model, $\left[n_{1}\right]+\left[n_{2}\right]=\left[n_{1}+n_{2}\right]$ and $\left[n_{1}\right] \cdot\left[n_{2}\right]=\left[n_{1} \cdot n_{2}\right]$, so the first two $\mathcal{R}^{\prime}$ axioms are trivially satisfied. For the third, it's immediate that if $n_{1} \neq n_{2}$ then $f \in \mathfrak{H}^{\omega, m}\left(\overline{n_{1}}=\overline{n_{2}}\right)$, hence $t \in \mathfrak{H}^{\omega, m}\left(\overline{n_{1}} \neq \overline{n_{2}}\right)$. For dealing with variables, we'll suppose we've fixed a variable assignment $\mathfrak{f}$. $(\mathcal{R} 5)$ is trivial, given that heap models are linearly ordered.

For $(\mathcal{R} 4)$, suppose that $t \in \mathfrak{H}^{\omega, m}(x \leq \bar{n})=\mathfrak{H}^{\omega, m}(\exists y(x+y=\bar{n}))$. Thus there is an $i$ in the domain of $\mathfrak{H}^{\omega, m}$ s.t. $\langle[x+y],[n]\rangle \in I_{\mathfrak{H}^{\omega, m}}^{+}[i / y](=)$, and so $\mathfrak{H}^{\omega, m}(x)+i \sim n$. It is clear that if either $\mathfrak{H}^{\omega, m}(x), i \geq \omega$ then this is false. Hence $\mathfrak{H}^{\omega, m}(x), i<\omega$, in which case $\mathfrak{H}^{\omega, m}(x)+i=n$. Since the finite part of $\mathfrak{H}^{\omega, m}$ is just the structure of $\mathbb{N}$, thus $\mathfrak{H}^{\omega, m}(x)$ must be one of the natural numbers less than $n$, hence $t \in \mathfrak{H}^{\omega, m}(x=\overline{0}) \cup \cdots \cup \mathfrak{H}^{\omega, m}(x=\bar{n}) \Longleftrightarrow t \in$ $\mathfrak{H}^{\omega, m}(x=\overline{0} \vee \cdots \vee x=\bar{n})$ as desired.

For ('), suppose that $t \in \mathfrak{H}^{\omega, m}(\neg(x=\overline{0} \vee \cdots \vee x=\bar{n}))$, and so $f \in$ $\mathfrak{H}^{\omega, m}(x=\overline{0}) \cap \cdots \cap \mathfrak{H}^{\omega, m}(x=\bar{n})$. Since $n<\omega, I_{\mathfrak{H}^{\omega, m}}(x) \neq 0, \ldots, n$, and thus for any $i$ in the domain of $\mathfrak{H}^{\omega, m}, \mathfrak{H}^{\omega, m}(x)+i \neq n$ and thus $t \in \mathfrak{H}^{\omega, m}[i / y](x+y \neq$ $\bar{n})$, hence $t \in \mathfrak{H}^{\omega, m}(\forall y(x+y \neq \bar{n}))=\mathfrak{H}^{\omega, m}(x \not \leq \bar{n})$, as desired.

From this and Lem. 3.11, it follows that:

Corollary 3.22 ( $\Sigma_{1}$-Completeness). If $A \in \Sigma_{1}$ and $N \vDash_{C L} A\left(t_{1}, \ldots, t_{n}\right)$ then $\mathfrak{H}_{\boldsymbol{A} 3}^{\omega, m} \vdash A\left(\bar{t}_{1}, \ldots, \bar{t}_{n}\right)$.

Which in turn entails that each $\mathfrak{H}^{\omega, m}$ is Rosser, in the sense defined above. Unfortunately, the argument format above can't be used here for the reason that no $\mathfrak{H}^{\omega, m}$ is $\Sigma_{1}$-consistent:

Proposition 3.23. For any $m \in \mathbb{N}$, there is an $A \in \Sigma_{1}$ s.t. $\mathfrak{H}_{\boldsymbol{L} P}^{\omega, m} \vdash \neg A \wedge A$.

Proof. For $m>0$, note that $\mathfrak{H}_{\mathbf{A} \mathbf{3}}^{\omega, m} \vdash \neg \exists x(x+\bar{m}<x) \wedge \exists x(x+\bar{m}<x)$. For $m=0$, note that $\mathfrak{H}_{\mathbf{A} 3}^{\omega, 0} \vdash \neg \exists x(x<x) \wedge \exists x(x<x)$.

This means that we can't simply use the [3] method, as it stands, to obtain undecidability; $\Sigma_{1}$-consistency is an essential ingredient, as displayed, for instance, in the proof that $\mathcal{R}_{\mathbf{A} 3}^{\prime}$ is undecidable. Having said that, there are reasons to think that such a method could be used. For while we may not have $\Sigma_{1}$-consistency, we may be able to find other consistent fragments of the theory which are appropriate to an undecidability proof along the lines 
given for $\mathcal{R}_{\mathbf{A} 3}^{\prime}$. In the rest of this section, I'll say a bit about how that might be done, before explicitly stating the salient open problems.

First off, we can more or less immediately pick out a consistent fragment as follows:

Definition 3.24. Call $A \in \mathcal{L}$ essentially numerical iff it is either is an identity obtaining between numerals or functions thereon, has the form $x \leq \bar{n}$ or $x<\bar{n}$, or is composed out of these by the connectives.

Note that essentially numerical formulas do not coincide with $\Delta_{0}$-formulas defined above for the reason that the latter were defined 'classically', however the point is basically that essentially numerical formulas are the ' $\Delta_{0}$-formulas according to A3.'

Lemma 3.25. If $A$ is essentially numerical then $\mathfrak{H}_{\boldsymbol{A} 3}^{\omega, m}(A) \neq\{t, f\}$.

Proof. First suppose that $\mathfrak{H}^{\omega, m}\left(\overline{n_{1}}=\overline{n_{2}}\right)=\{t, f\}$, so then we have $n_{1} \sim n_{2}$ and $n_{1} \neq n_{2}$, which is impossible since $n_{1}, n_{2}<\omega$. Since $\sim$ is a congruence w.r.t. $S,+, \cdot$ the result holds if we also consider formulas in which these symbols are applied to numerals. Next, suppose that $\mathfrak{H}^{\omega, m}(\exists y(x+y=\bar{n}))=$ $\{t, f\}$, so there are $i, j$ such that $\mathfrak{H}^{\omega, m}[i / x, j / y](x+y=\bar{n})=\{t, f\}$ and thus $[i+j]=[n]$ and $i+j \neq n .[n]=\{n\}$ since $n<\omega$, and therefore $[i+j]=[n]$ holds iff $i+j=n$, so this is also impossible. Once again, congruentiality of $\sim$ means that this also holds for complex $\bar{n}$. It is immediate that if $B, C$ only take values in $\{\{t\},\{f\}\}$, then so do $\neg B, \neg C$ and $B * C$ for $* \in\{\wedge, \vee, \rightarrow\}$.

This lemma expresses the fact that the standard number line, which forms an initial segment of $\mathfrak{H}^{\omega, m}$, behaves classically. There may be some trickery available to marshal this fact in order to get around the fact that $\mathfrak{H}_{\mathrm{A3}}^{\omega, m}$ is $\Sigma_{1}$-inconsistent. For instance, we know, from $\Sigma_{1}$-completeness, that there is a $\Sigma_{1}$-formula $A(x)$ such that $n \in \alpha \Rightarrow \mathfrak{H}_{\mathbf{A} 3}^{\omega, m} \vdash A(\bar{n})$ and also $n \in \beta \Rightarrow$ $\mathfrak{H}_{\mathbf{A} 3}^{\omega, m} \vdash \neg A(\bar{n})$. The difficulty is that we have no guarantee that, in the first case, we may not also have $\mathfrak{H}_{\mathbf{A} 3}^{\omega, m} \vdash \neg A(\bar{n})$, and in the second $\mathfrak{H}_{\mathbf{A} 3}^{\omega, m} \vdash A(\bar{n})$. So one possible trick would involve showing that there is a recursive way of constructing, from $A(\bar{n})$ a formula $A_{n}$ which witnesses the separation, but where $\mathfrak{H}_{\mathbf{A} 3}^{\omega, m} \nvdash A_{n} \wedge \neg A_{n}$. I'll leave the question of finding such a trick here, and instead just pose the salient problem.

Open Problem 3.26. Is $\mathfrak{H}_{\boldsymbol{A} 3}^{\omega, m}$ undecidable?

Australasian Journal of Logic (18:5) 2021, Article no. 11 
I suspect the answer is yes. The reason, not even remotely decisive, can be illustrated by a mental picture. As we proceed from $\mathfrak{H}^{1, m}$ to heap models with longer and longer tails, we seem to be pushing back the point at which we first meet an inconsistent number, and hence including a larger and larger finite initial segment of the natural number line. ${ }^{11}$ So when we reach $\mathfrak{H}^{\omega, m}$, we've got the whole, consistent, standard number line, and the only inconsistencies come in after the point of infinity - so long as we can find the information of classical arithmetic in some consistent fragment of the resulting theory, then it seems likely that we can exploit it to provide an undecidability argument, as gestured at above.

Of course this is just a concrete instance of a further open problem, which is to show that there are undecidable, inconsistent extensions of $\mathcal{R}_{\mathbf{A} 3}^{\prime}$. It seems likely that there are ways of building such a theory by adding some formulas contradicting theorems of $\mathcal{R}_{\mathbf{A} 3}^{\prime}$, but the more interesting question is whether there are reasonably natural inconsistent, undecidable extensions and I would take $\Sigma_{1}$-inconsistency and having a detaching conditional to be desiderata of reasonable naturalness. In light of this, I'll pose the following more general problem, to which, to my knowledge, there is no answer as yet. ${ }^{12}$

Open Problem 3.27. Are there any $\Sigma_{1}$-inconsistent, undecidable arithmetic theories in $\boldsymbol{A} 3, \boldsymbol{R M} 3, \boldsymbol{R}$, or any other strong paraconsistent logic?

\section{Appendix: Hilbert Systems for A3 and RM3}

The logics of interest here share in common the following basic axioms:

(Id) $A \rightarrow A$

(B) $(A \rightarrow B) \rightarrow((C \rightarrow A) \rightarrow(C \rightarrow B))$

(Cl) $A \rightarrow((A \rightarrow B) \rightarrow B)$

(WI) $(A \rightarrow B) \wedge A \rightarrow B$

$(\wedge \mathbf{E}) A \wedge B \rightarrow A, A \wedge B \rightarrow B$

\footnotetext{
${ }^{11}$ For some related philosophical discussion, see [20].

${ }^{12}$ The problem concerning the decidability of $\mathbf{R M} \omega$, posed by Mortensen [16, p.31], while not an instance of this question, is closely related and is, to my knowledge, as of yet unsolved. $\mathbf{R M} \omega$ is the intersection of various cyclic models, interpreted in various oddly-many valued extensions of $\mathbf{R M}$ - where $\mathbf{R M} 3$ is the three-valued among these.
}

Australasian Journal of Logic (18:5) 2021, Article no. 11 
$(\wedge \mathbf{I})(A \rightarrow B) \wedge(A \rightarrow C) \rightarrow(A \rightarrow B \wedge C)$

$(\vee \mathbf{E}) A \rightarrow A \vee B, B \rightarrow A \vee B$

$(\vee \mathbf{I})(A \rightarrow C) \wedge(B \rightarrow C) \rightarrow(A \vee B \rightarrow C)$

(Dist) $A \wedge(B \vee C) \rightarrow(A \vee B) \wedge(A \vee C)$

(DNE) $A \leftrightarrow \neg \neg A$

$\left(\mathrm{DeM}_{1}\right) \neg(A \wedge B) \leftrightarrow \neg A \vee \neg B$

$\left(\mathrm{DeM}_{2}\right) \neg(A \vee B) \leftrightarrow \neg A \wedge \neg B$

(LEM) $A \vee \neg A$

and the following rules (where $\Rightarrow$ is a separator indicating a rule of proof, in Smiley's sense [25]):

$(\mathrm{rMP}) A \rightarrow B, A \Rightarrow B$

(rADJ) $A, B \Rightarrow A \wedge B$

To obtain A3 we add to this set of basic axioms the following three:

(K) $A \rightarrow(B \rightarrow A)$

(Peirce) $((A \rightarrow B) \rightarrow A) \rightarrow A$

(Cntr) $\neg(A \rightarrow B) \leftrightarrow(A \wedge \neg B)$

To obtain $\mathbf{R M} 3$ we add to the basic axioms the following three (for $\mathbf{R}$ just add the first of these):

(Cont) $(A \rightarrow B) \rightarrow(\neg B \rightarrow \neg A)$

(Mingle) $A \rightarrow(A \rightarrow A)$

(3) $A \vee(A \rightarrow B)$

The following axioms (and rule) are adequate for the first-order extensions of these propositional logics (some are redundant in one or the other system):

$(\forall \mathbf{E}) \forall x A(x) \rightarrow A(y)$

$(\forall \mathbf{I}) \forall x(A \rightarrow B(x)) \rightarrow(A \rightarrow \forall x B(x))$

$(\forall \vee) \forall x(A \vee B(x)) \rightarrow(A \vee \forall x B(x))$ where $y$ free for $x$ in $A$. where $x$ not free in $A$. where $x$ not free in $A$.

Australasian Journal of Logic (18:5) 2021, Article no. 11 
$(\forall \wedge) \forall x A(x) \wedge \forall x B(x) \rightarrow \forall x(A(x) \wedge B(x))$

$(\exists \wedge) \quad A \wedge \exists x B(x) \rightarrow \exists x(A \wedge B(x))$

where $x$ not free in $A$.

$(\exists \mathbf{I}) \quad A(y) \rightarrow \exists x A(x)$

$(\exists \mathbf{E}) \forall x(B(x) \rightarrow A) \rightarrow(\exists x B(x) \rightarrow A)$

$x$ not free in $A$.

$\left(Q D_{1}\right) \forall x \neg A(x) \leftrightarrow \neg \exists x A(x)$

$\left(Q D_{2}\right) \exists x \neg A(x) \leftrightarrow \neg \forall x A(x)$

(rGen) $A y \Rightarrow \forall x A(x)$

Soundness and completeness theorems are standard, but that for A3 may be found in [27].

\section{Acknowledgments}

Thanks are due to audience at the meeting, held at UNAM, commemorating the 50th anniversary of the Logician's Liberation League, as well as those at talks given to the Group in Pure and Applied Logic at Kansas State University and the Applied Mathematical Logic seminar at the Institute of Computer Science of the Czech Academy of Sciences, for discussion of early versions. Furthermore, I'd like to thank Shay Allen Logan, Teresa Kouri Kissel, and a referee for very helpful commentary on a draft. Finally, I'm indebted to Eno Agolli for translating some parts of [24].

\section{References}

[1] A. R. Anderson, N. D. Belnap, and J. M. Dunn. Entailment, The Logic of Relevance and Necessity, Vol. II, Princeton University Press, Princeton, 1992.

[2] A. Avron. Natural 3-valued logics: Characterisation and proof theory. Journal of Symbolic Logic, 49:334-342, 1991.

[3] G. Badia, P. Cintula, P. Hájek, and A. Tedder. How Much Propositional Logic Suffices for Rosser's Undecidability Theorem? The Review of Symbolic Logic, (Forthcoming).

Australasian Journal of Logic (18:5) 2021, Article no. 11 
[4] I. D'Ottaviano and N. C. A. da Costa. Sur un probléme de Jaśkowski. Comptes Rendus de l'Académie des Sciences de Paris, 270:1349-1353, 1970.

[5] J. M. Dunn. Relevant Robinson's Arithmetic. Studia Logica, 38(4):407418, 1979.

[6] J. M. Dunn. A theorem in 3-valued model theory with connections to number theory, type theory, and relevant logic. Studia Logica, 38:149169, 1979.

[7] T. M. Ferguson. Variations on the Collapsing Lemma. In C. Başkent and T. M. Ferguson, editors, Graham Priest on Dialetheism and Paraconsistency, pages 249-270. Springer, Switzerland, 2020.

[8] H. Friedman and R. K. Meyer. Whither relevant arithmetic? The Journal of Symbolic Logic, 57(3):824-831, 1992.

[9] A. P. Hazen and F. J. Pelletier. K3, Ł3, LP, RM3, A3, FDE, M: How to make many-valued logics work for you. In H. Omori and H. Wansing, editors, New Essays on Belnap-Dunn Logic, pages 155-190. Springer, Switzerland, 2020.

[10] R. Kaye. Models of Peano Arithmetic. Oxford University Press, Oxford, 1991.

[11] R. K. Meyer. Relevant Arithmetic. Bulletin of the Section of Logic of the Polish Academy of Sciences, 5:133-137, 1976.

[12] R. K. Meyer. The Consistency of Arithmetic. The Australasian Journal of Logic 18(5):288-378

[13] R. K. Meyer Arithmetic Formulated Relevantly. The Australasian Journal of Logic !8(5):153-287

[14] R. K. Meyer and C. Mortensen. Inconsistent models for relevant arithmetics. The Journal of Symbolic Logic, 49(3):917-929, 1984.

[15] R. K. Meyer and C. Mortensen. Alien Intruders in Relevant Arithmetic. Technical Report TR-ARP-9/87, Australian National University, 1987. Reprinted in The Australasian Journal of Logic 18(5):400-421

[16] C. Mortensen. Inconsistent Mathematics. Mathematics and Its Applications Series, Kluwer Academic Publishers, 1995.

[17] J. B. Paris and N. Pathmanathan. A note on Priest's finite inconsistent arithmetics. Journal of Philosophical Logic, 35:529-537, 2006.

[18] J. B. Paris and A. Sirokofskich. On LP-models of arithmetic. Journal of Symbolic Logic, 73(1):212-226, 2008.

[19] G. Priest. The Logic of Paradox. Journal of Philosophical Logic, 8(1):219-241, 1979.

Australasian Journal of Logic (18:5) 2021, Article no. 11 
[20] G. Priest. Is arithmetic consistent? Mind, 103(411):337-349, 1994.

[21] G. Priest. Inconsistent models of arithmetic. Part I: Finite models. Journal of Philosophical Logic, 26(2):223-235, 1997.

[22] G. Priest. Inconsistent models of arithmetic. Part II: The general case. The Journal of Symbolic Logic, 65(4):1519-1529, 2000.

[23] G. Priest. An Introduction to Non-Classical Logic: From If to Is, 2nd Ed. Cambridge University Press, Cambridge, 2008.

[24] A. Sirokofskich. Model Theory, with an Emphasis on Arithmetic (original in Greek) PhD Dissertation, National and Kapodistrian University of Athens, 2007.

[25] T. Smiley. Relative necessity. Journal of Symbolic Logic, 28(2):113-134, 1963.

[26] A. Tarski, A. Mostowski, and R. Robinson. Undecidable Theories, North-Holland, 1953.

[27] A. Tedder. Paraconsistent Logic for Dialethic Arithmetics M.A. Thesis, University of Alberta, 2014.

[28] A. Tedder. Axioms for finite collapse models of arithmetic. The Review of Symbolic Logic, 8(3):529-539, 2015.

Australasian Journal of Logic (18:5) 2021, Article no. 11 